\title{
Predictors of breast self-examination behavior in housewives based on trans-theoretical model
}

\author{
Mitra Moodi $^{1}$ (D), Mohammad Reza Miri ${ }^{1}(\mathbb{D})$, Gholamreza Sharifzadeh $^{1}$ (i), \\ $\underline{\text { Maryam Miri }^{2}}$, Ensiyeh Norozi ${ }^{1}(\mathbb{D})$, Soodabeh Es-haghi ${ }^{3}(\mathrm{D})$ \\ ${ }^{1}$ Social Determinants of Health Research Center, Birjand University of Medical Sciences, Birjand, Iran. \\ ${ }^{2}$ Corresponding author; Department of Health Education and Promotion, Social Determinants of Health Research Center, Birjand \\ University of Medical Sciences, Birjand, Iran. \\ Tel: +989157211385_E-mail:mmiri1062@gmail.com \\ ${ }^{3}$ General practitioner, Social Determinants of Health Research Center, Birjand University of Medical Sciences, Birjand, Iran.
}

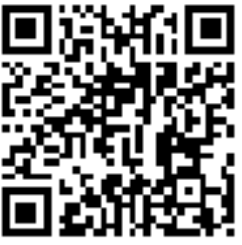

Citation Moodi M, Miri MR, Sharifzadeh Gh, Miri M, Norozi E, Es-haghi S. [Predictors of breast selfexamination behavior in housewives based on trans-theoretical model]. J Birjand Univ Med Sci. 2019; 26(1):64-73. [Persian]

DOI http://doi.org/10.32592/JBirjandUnivMedSci.2019.26.1.107

Received: May 27, 2018

Accepted: February 4, 2019

\begin{abstract}
Background and Aim: Breast cancer is the second leading cause of death from cancer among women. Monthly breast SelfExamination is an important screening strategy for early detection of breast cancer, but few women do this. The purpose of this study was to determine predictors of breast self-examination behavior in housewives based on the trans-theoretical model.

Materials and Methods: This descriptive-analytic study was incorporated 450 housewives aged between 20 to 40 years old referring to health centers in Birjand. Sampling was through the Stratified Sampling method. Data collection was carried out through a researcher-made questionnaire including demographic information and dimensions of the meta-theory model and knowledge questions whose validity and reliability were confirmed. The data were analyzed by SPSS 19 software and analyzed by multivariate logistic regression and Pearson correlation coefficient test.
\end{abstract}

Results: Mean age of the housewives was $30.75 \pm 5.24$ years. As regarding the stages of transition in breast self-examination (BSE) behavior, $32.9 \%$ were in the pre-contemplation stage, $19.6 \%$ in contemplation, $23.3 \%$ in preparedness, $18.2 \%$ in action, and $6 \%$ in maintenance stages. Regression analysis showed that self-efficacy, knowledge, and education level were predictors of BSE.

Conclusion: The findings of the study showed that self-efficacy and knowledge were predictors of BSE. Therefore, BSE behavior can be reinforced via educational programs that aim at increasing awareness and self-efficacy among women.

Key Words: Transtheoretical Model; Breast Cancer; Breast Self-Examination; Predictor 


\title{
يشيشويى كنندهاى رفتار خودآزمايى بستان در زنان خانهدار، بر اساس مدل فر ونظرى
}

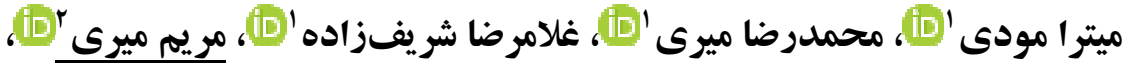

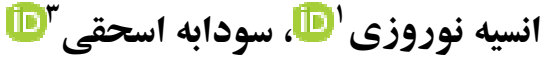

\section{جكيده}

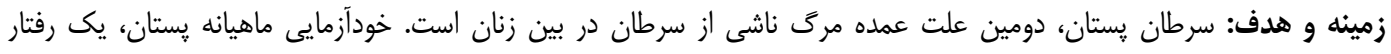

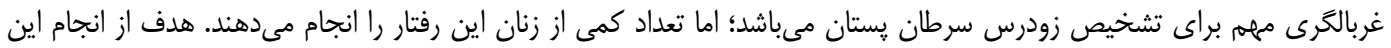

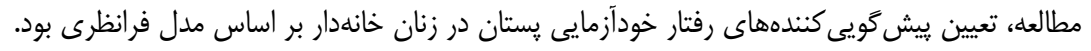

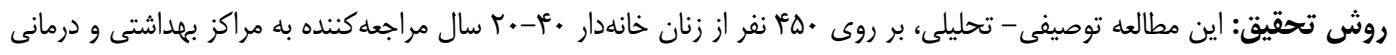

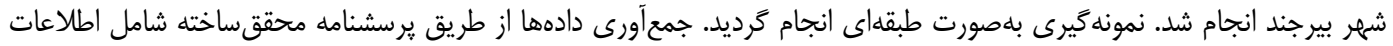

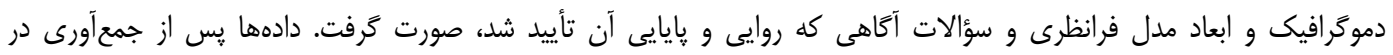

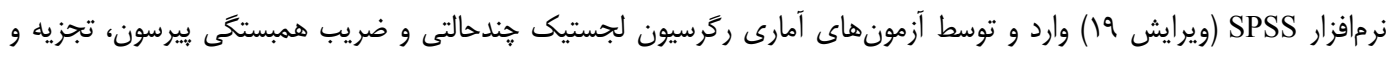
تحليل شد.

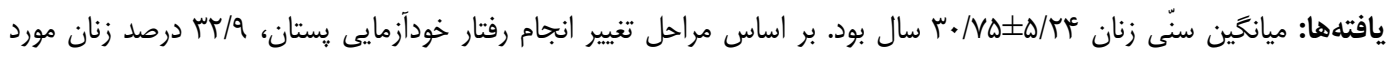

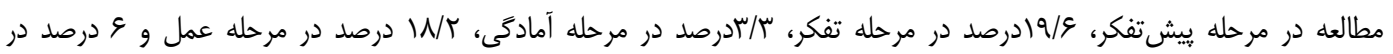

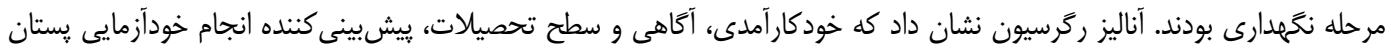

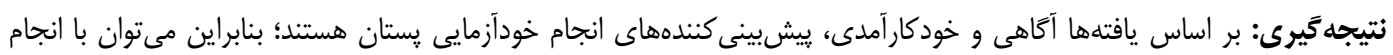

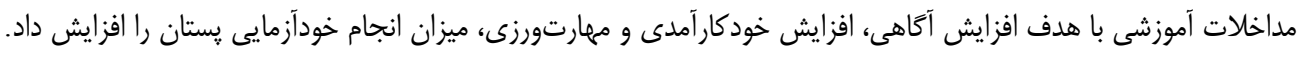

$$
\begin{aligned}
& \text { وازههاى كليدى: مدل فرانظرى؛ سرطان پستان؛ خودآزمايى پستان؛ ييش گويى كننده }
\end{aligned}
$$

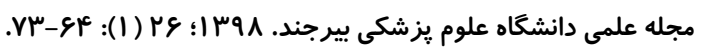

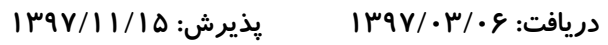

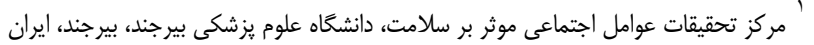

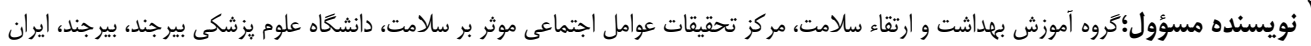

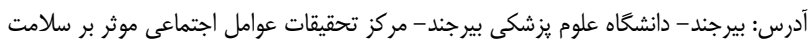

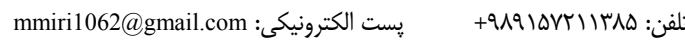
"يزشك عمومى، مركز تحقيقات عوامل اجتماعى موثر بر سلامت، دانشكاه علوم بزشكى بيرجنله، بيرجنه ايران 


$$
\text { كافى انجام نمىشود (·) (1). }
$$

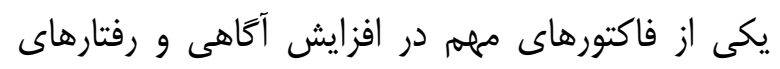

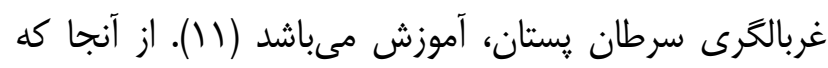

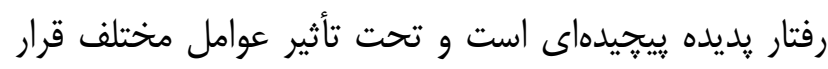

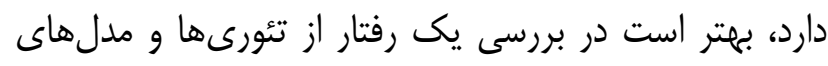

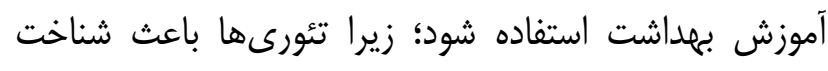

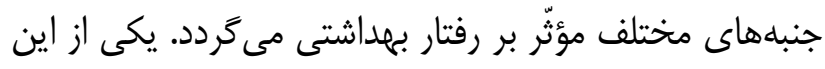

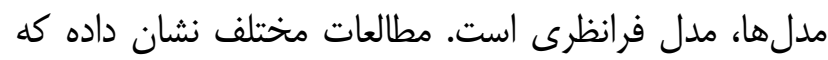

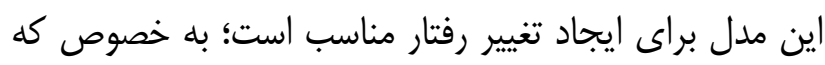

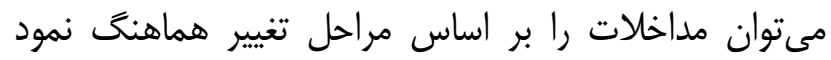

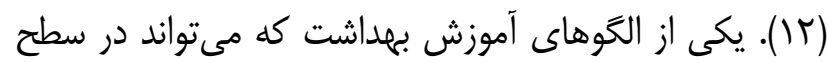

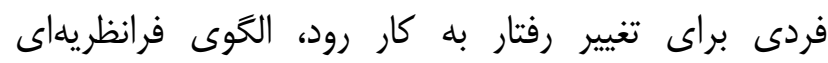

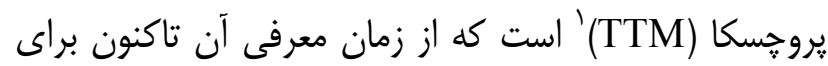
طيف وسيعى از رفتارهاى سلامتى استفاده شده است.

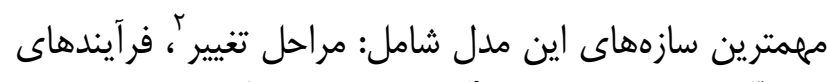

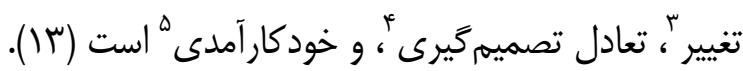

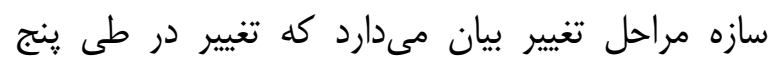

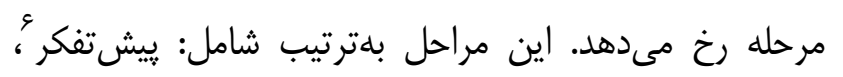

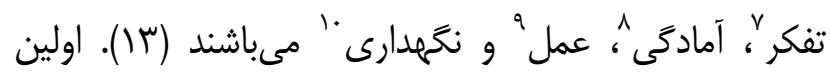

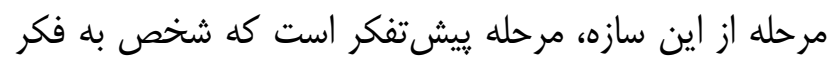

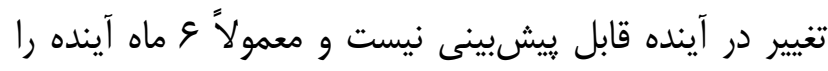

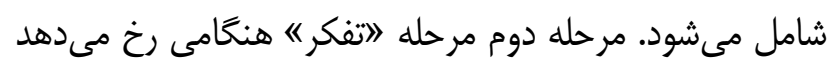

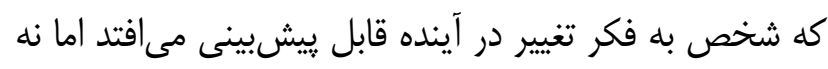

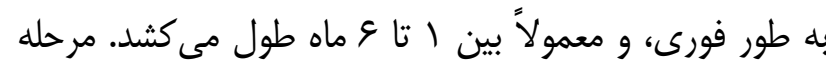

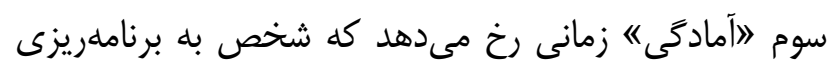

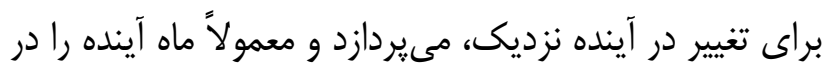

\footnotetext{
${ }^{1}$ Trans Theoritical Model

2 Stage of Change

3 Processes of change

${ }^{4}$ Decision making balance

${ }^{5}$ Self efficacy

${ }^{6}$ Precontemplation

${ }^{7}$ Contemplation

${ }^{8}$ Preparation

${ }^{9}$ Action

${ }^{10}$ Maintenance
}

مقدمه سرطان يستان، دومين سرطان شايع در بين زنان است

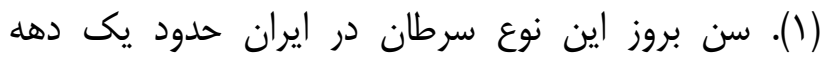

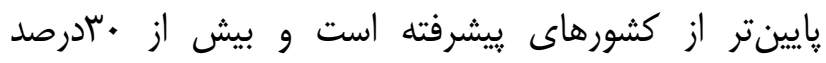

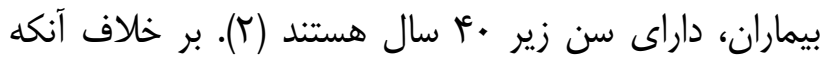

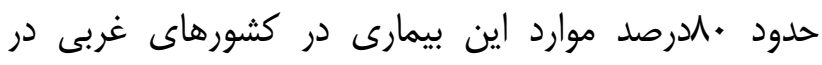

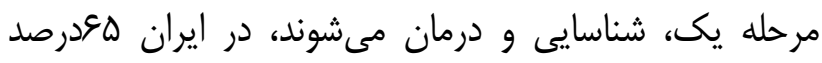

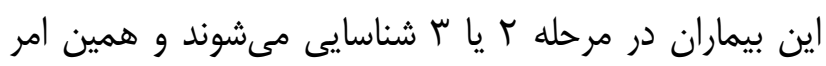

درمان قطعى اين بيماران را با مشكل مواجه مى كند (َّ).

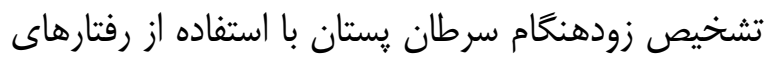

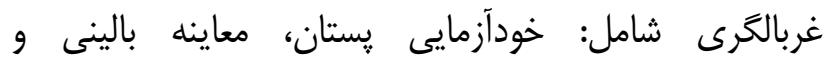

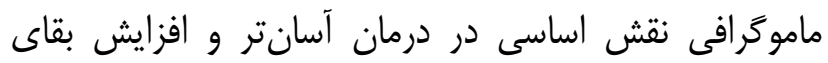

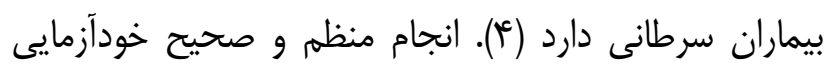
يستان يك روش ساده، اقتصادى، آسان و بیىضرر است كه آنه مىتواند بدون تجهيزات در حالتى كه از حريم خصوصى زن رنان

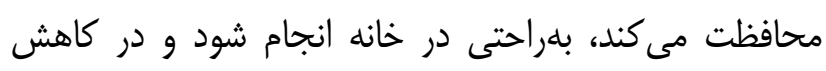

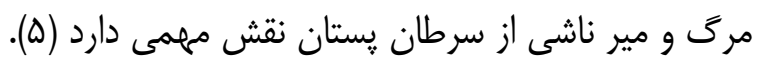

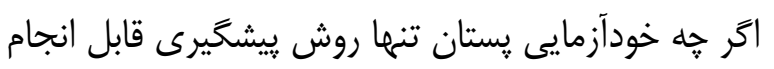

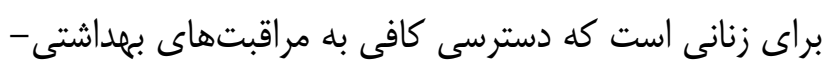

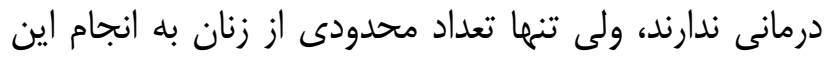

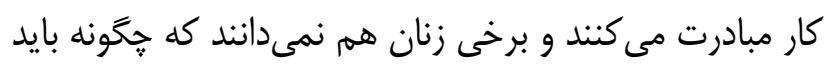

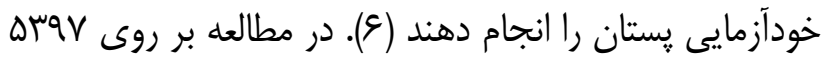

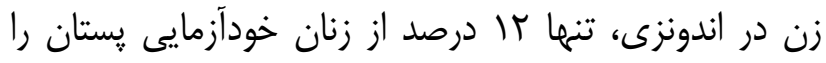

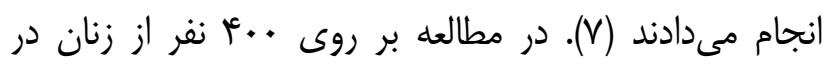

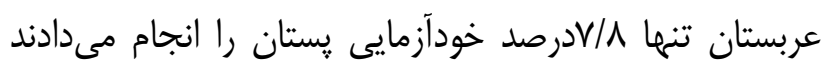
(^)

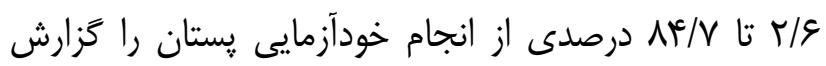

$$
\text { كردهاند (9). }
$$

عوامل مختلفى ممكن است در انجام يا عدم انجام

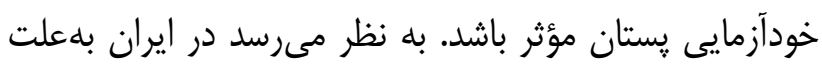

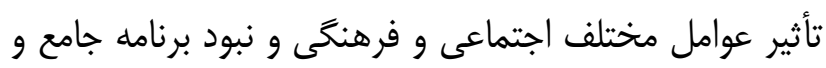

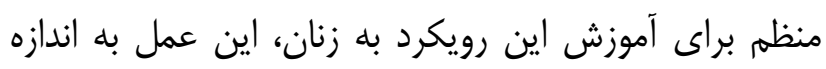


برآورد گرديد. نمونهَيرى بلهروش طبقهاى' صورت گرفت.

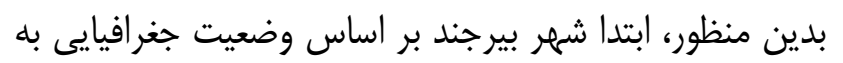

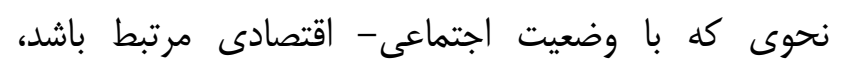

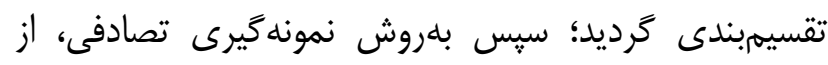
يايعاههاى بهراشتى تحت يوشش هر منطقه دو پايخاه انتخاب

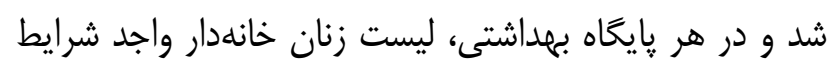

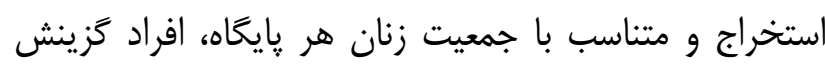

شدند.

معيارهاى ورود ببه مطالعه شامل: زنان خانهدار •r-r-r سال مراجعهكننده به مراكز بهداشتى و درمانى بدون سابقه ونهان

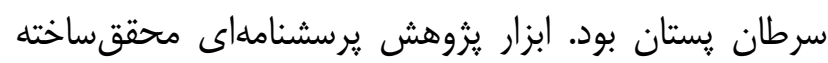

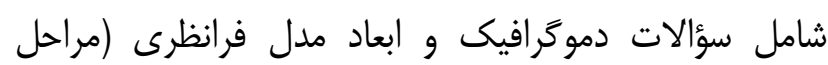

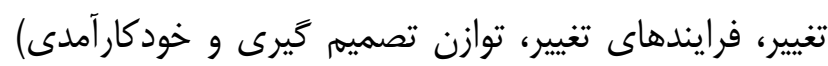

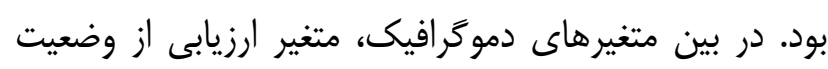

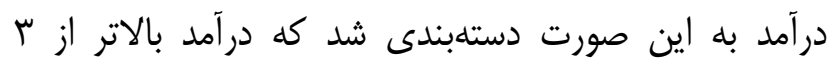

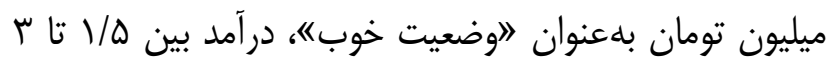

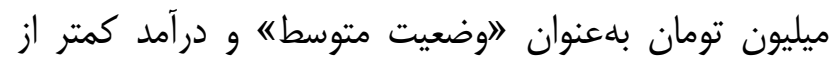

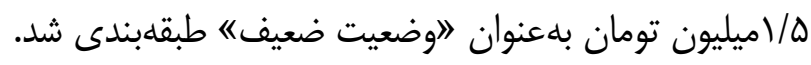

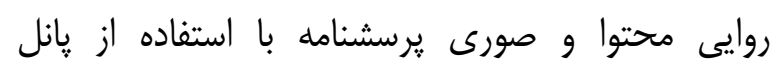

خبر كان تأييد شد؛ بدين ترتيب كه در ابتدا با مطالعه مقالات

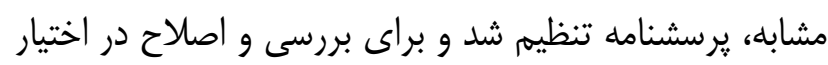
•ا نفر از متخصصين و صاحبنظران آموزش بهداشت (سنفر)،

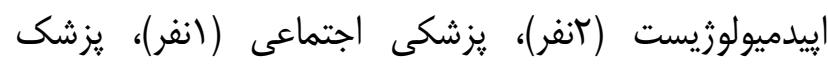

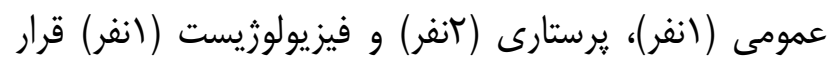
كرفت. شاخص روايى محتوا (CVI)

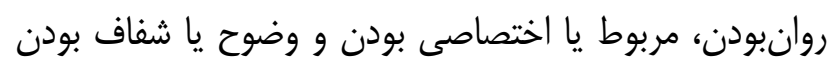

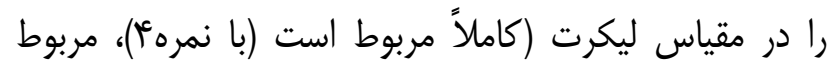

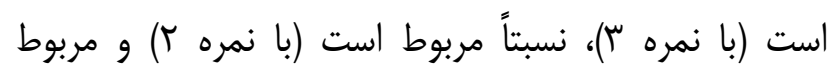
نيست (با نمرها) محاسبه مى كند. شاخص روايى محتوا

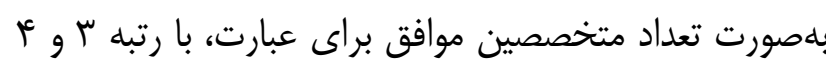

\footnotetext{
${ }^{1}$ Stratified Sampling

${ }^{2}$ Content validity index
}

بر مى گيرد. مرحله جهارم مرحله "اعمل" است كه در آن

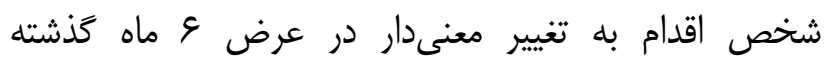

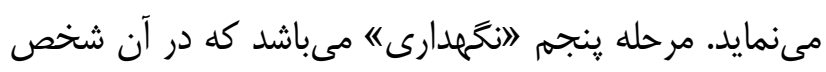
به حفظ رفتار براى دورهاى از زمان مىيردازد و معمولاً

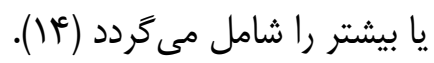

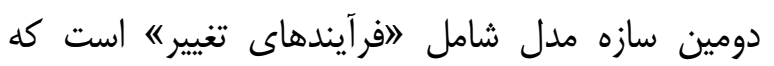

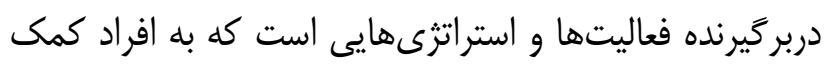

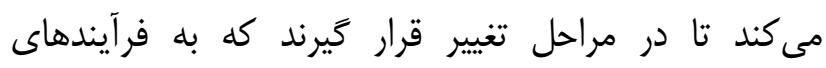

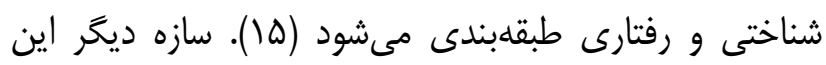

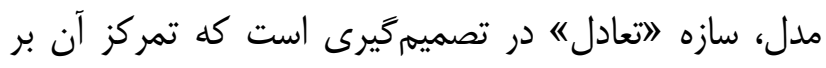

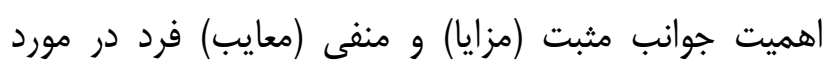

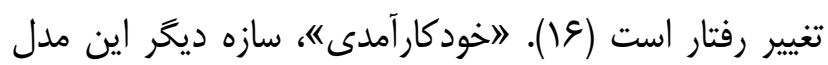

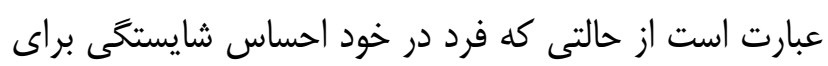
انجامدادن كارى با اطمينان به توانايى خود براى انجام عملى درد دارد (IV) ( ) - (IV)

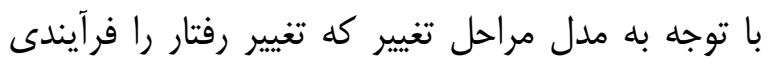
مرحلهاى در نظر مى

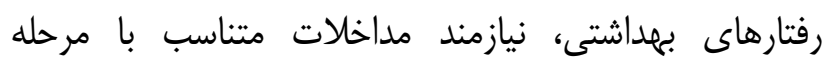

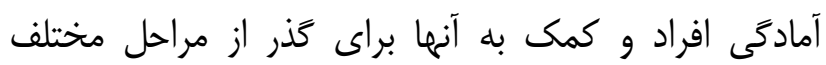

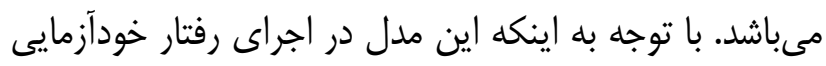

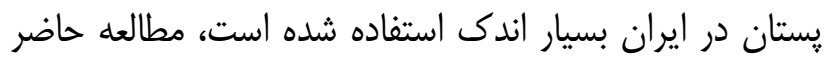

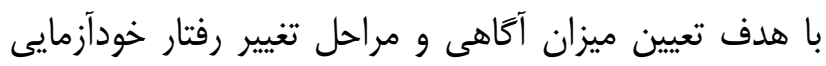

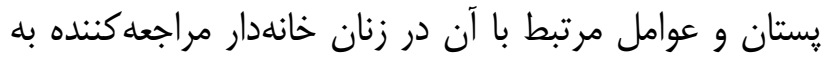

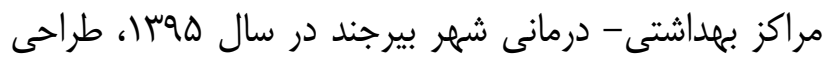

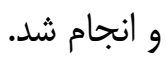

\section{روش تحقيق}

اين مطالعه توصيفى - تحليلى، بر روى • لهأ نفر از زنان

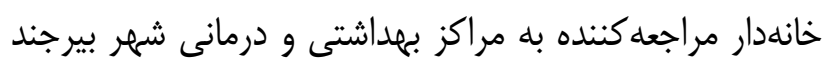

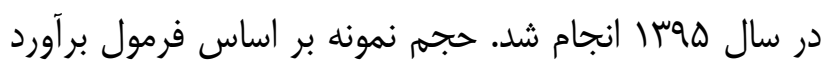

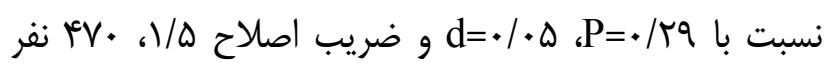


زياد) با نمره ا تا ه ياسخ داده شد و حداقل نمره كسبشده

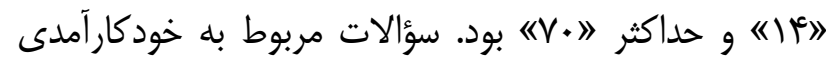

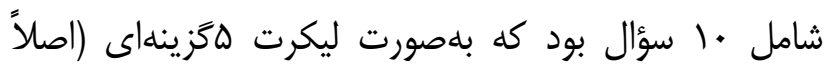

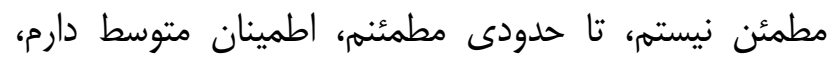

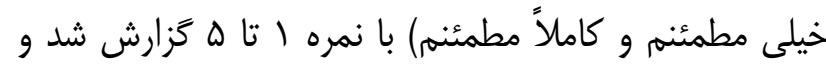

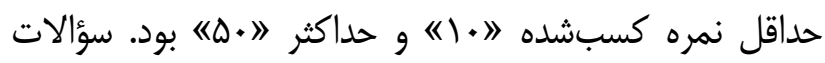

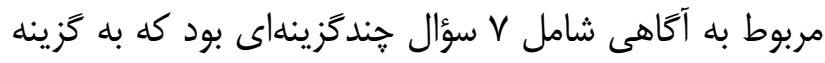

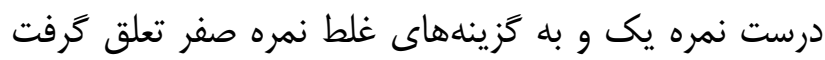

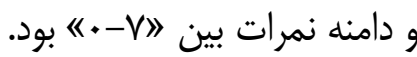

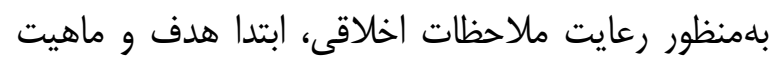

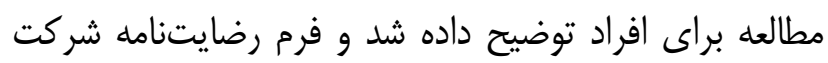

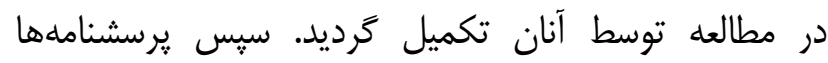

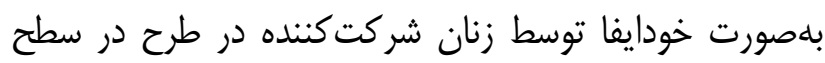

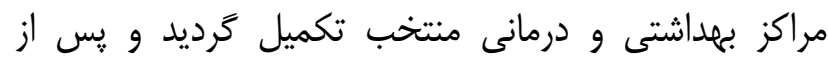

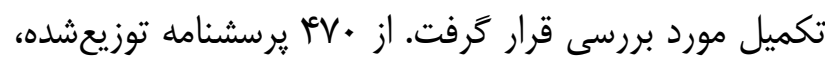

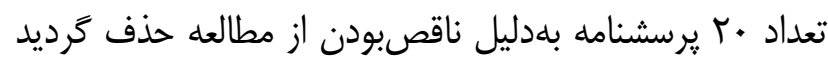

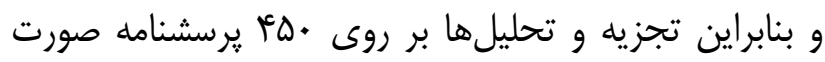

بلمنظور تجزيه و تحليل، دادهها در نرمافزار SPSS (ويرايش 19) وارد گرديد. ابتدا شاخصهائ دهاى توصيفى شامل:

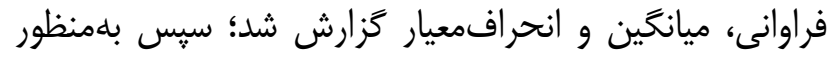
بررسى ارتباط سازههاى مدل فرين وانظرى (مراحل تغيير، توازن

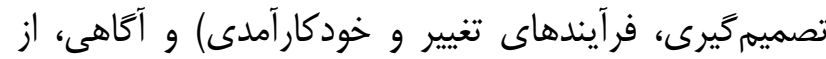

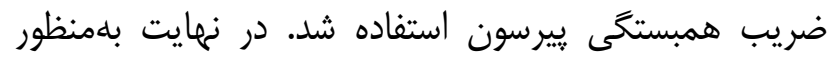

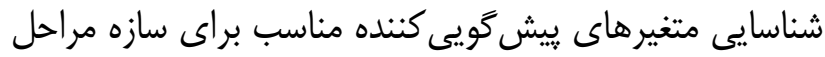

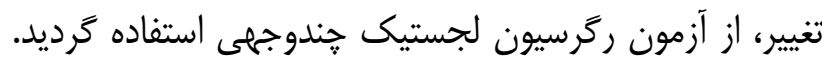
سطح معنىدارى ه درصد در نظر كرفته شد. اين مقاله

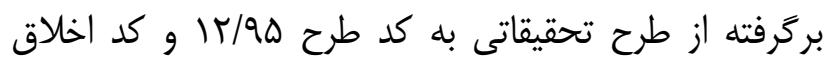
Ir.bums.1395.51

\section{يافتهها}

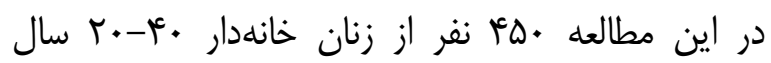

تقسيه بر تعداد كل متخصصان محاسبه شد. در صورتى كه

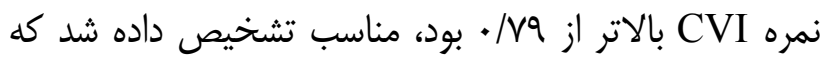

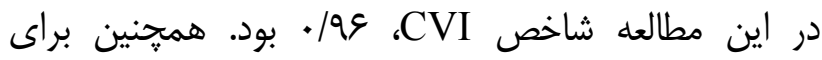

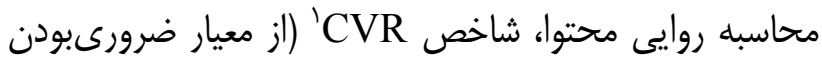
با عبارات: ضرورى است، مفيد است ولى ضرورى محتى نيست و

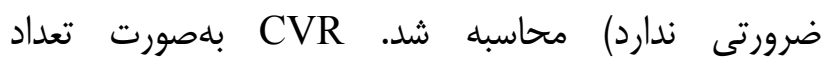
متخصصينى كه گزينه ضرورى را انتخاب كرده بودند، منهاى نداس نصف كل ارزيابىها تقسيه بر نصف كل ارزيابىها محاسبه

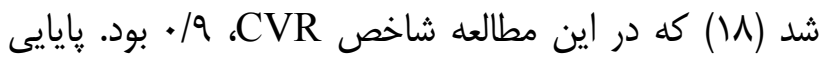

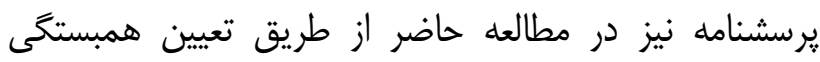

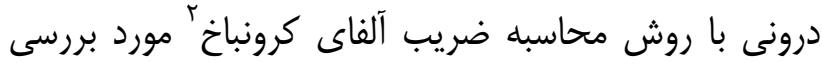

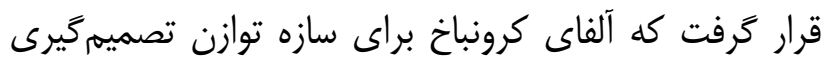

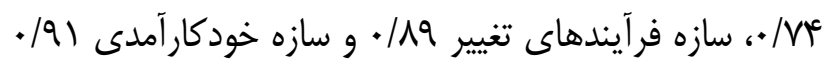

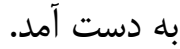
سؤالات مربوط به مراحل تغيير شامل يك سؤال

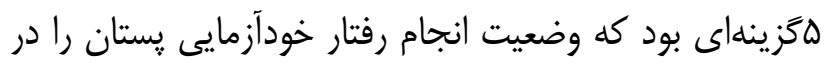

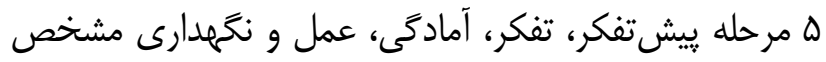

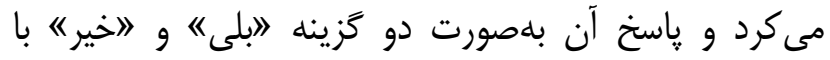

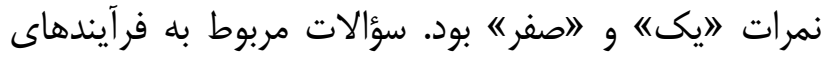

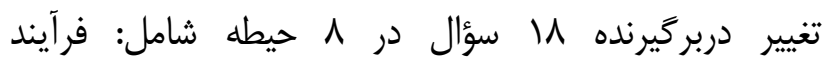

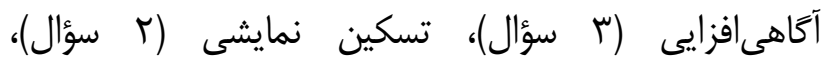

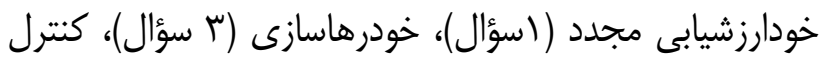

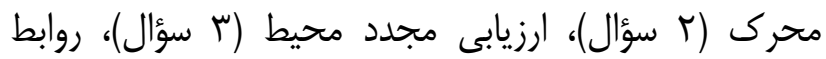

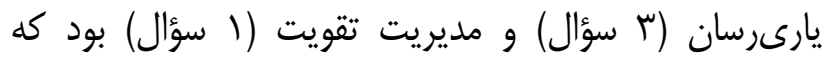

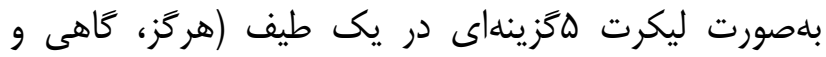

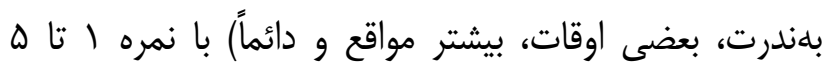

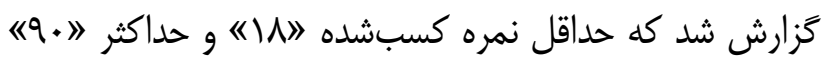

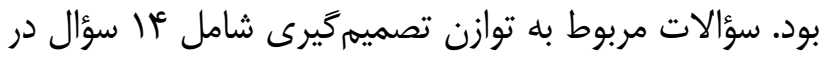

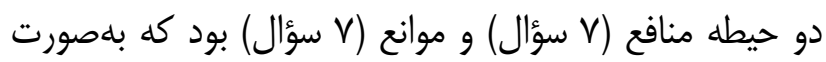
ليكرت هزَينهاى (به هيج وجله، كمى، متوسط، زياد و و خيلى

\footnotetext{
${ }^{1}$ Content validity ratio

${ }^{2}$ Cronbach s alpha
} 


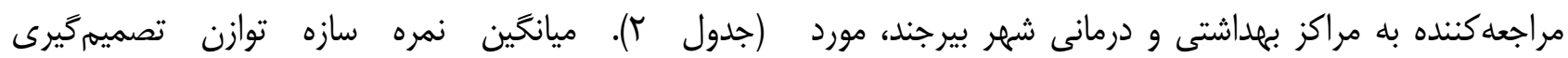

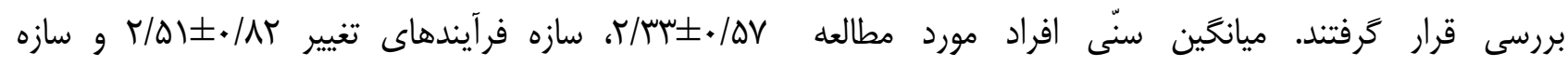

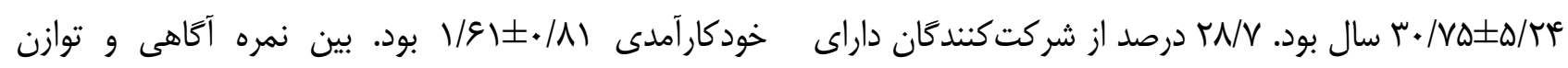

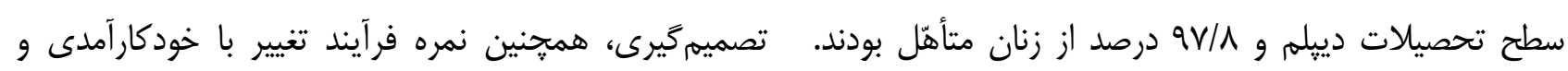

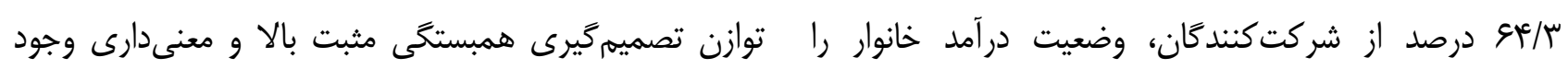

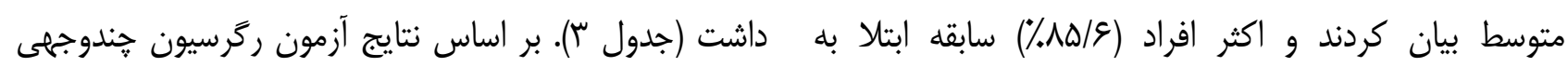
ناراحتى در يستان نداشتند (جدول () ).

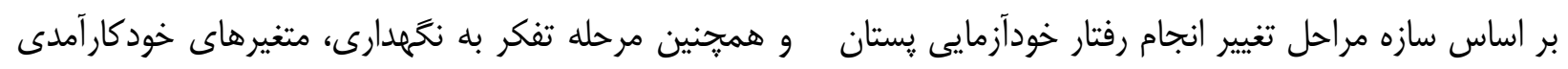

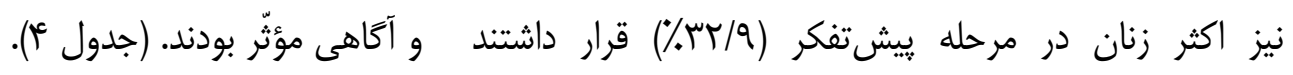
جدول ا - توزيع فراوانى متغيرهاى جمعيتشناختى در زنان مورد مطالعه

\begin{tabular}{|c|c|c|}
\hline فراوانى (درصد) & \multirow{3}{*}{\multicolumn{2}{|c|}{ زير •• سال •س سال }} \\
\hline$(r q / I)$ rrI & & \\
\hline$(0 . / 9)$ rrq & & \\
\hline$(r \Delta / \Lambda) \| G$ & ابتدايى & \\
\hline$(I V / T) \vee \Lambda$ & راهنمايى و دبيرستان & \\
\hline$(T N / V) ו K q$ & دييلم & سطح تحصيلات \\
\hline 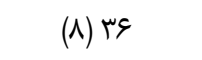 & فوق ديبله & \\
\hline$(r \cdot / T) 91$ & ليسانس و بالاتر & \\
\hline$(9 V / \Lambda) F F$. & متأهّل & م. . ت.أسّا \\
\hline$(T / T) 1$ & 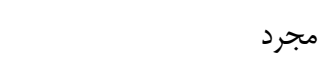 & عيت تاهل \\
\hline$(1 \cdot / \Delta) \& V$ & 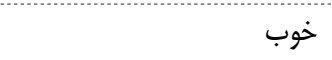 & \\
\hline$(g / / \mu)$ r & 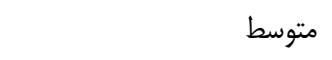 & ارزيابى از وضعيت درآمد خانوار \\
\hline$(T \Delta / T) \| T$ & ضعيف & \\
\hline$(9 / 1)+1$ & بلى و مراجعه به يزشك & \\
\hline$\left(\Delta / r^{\prime}\right) r{ }^{c}$ & بلى و بدون مراجعه به يزشك & سابقه ابتلا به ناراحتى پِتان در فرد \\
\hline$(\wedge ৯ / \varepsilon) \sqcap \wedge \Delta$ & خير بدون ناراحتى & \\
\hline$(\% / r) 19$ & بلى & سابقه ابتلا به سرطان پستان در \\
\hline 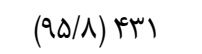 & 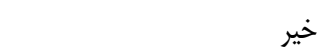 & بستخان درجه يك \\
\hline
\end{tabular}

جدول r- توزيع فراوانى مراحل تغيير انجام خودآزمايى يستان در زنان مورد مطالعه

\begin{tabular}{|c|c|c|}
\hline درصد & فراوانى & مراحل تغيير \\
\hline$r r / q$ & 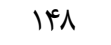 & يبشتفكر \\
\hline $19 / 8$ & $M$ & تفكر \\
\hline r & 1.0 & آمادگى \\
\hline$N / T$ & NT & عمل \\
\hline 9 & TV & نكمهدارى \\
\hline$\cdot$ & $\cdot$ & بركشت \\
\hline $1 .$. & in. & جمع \\
\hline
\end{tabular}


جدول ب- بر رسى ارتباط سازههاى مدل فر انظرى در زنان مورد مطالعه

\begin{tabular}{|c|c|c|c|c|}
\hline خودكارآمدى & فرآيندهاى تغيير & توازن تصميمَيرى & آنَاهى & متغير \\
\hline$r=\bullet / \cdot r$ & $\mathrm{r}=\cdot / 4 \varepsilon$ & $r=\cdot / r r$ & \multirow{2}{*}{1} & \multirow{2}{*}{ آكَاهى } \\
\hline $\mathrm{P}=\cdot / 4 q$ & $\mathrm{P}<\cdot / \ldots 1$ & $\mathrm{P}<\cdot / \cdots 1$ & & \\
\hline $\mathrm{r}=\cdot / \mu \mathrm{r}$ & $\mathrm{r}=\cdot|9|$ & \multirow[b]{2}{*}{1} & \multirow{2}{*}{---} & \multirow{2}{*}{ وازن تصميمَيرى } \\
\hline $\mathrm{P}<. / \ldots 1$ & $\mathrm{P}<\cdot / \ldots 1$ & & & \\
\hline $\begin{array}{c}\mathrm{r}=. \mid 9 \gamma^{\mathrm{C}} \\
\mathrm{P}<\cdot / . \cdot 1\end{array}$ & 1 & --- & --- & فرآيندهاى تغيير \\
\hline 1 & -- & -- & -- & خودكارآمدى \\
\hline
\end{tabular}

نتايج آزمون ضريب همبستخى ييرسون نشان داد كه بين نمره آَكاهى و توازن تصميم گيرى همبستخى مثبت و معنى دارى وجود

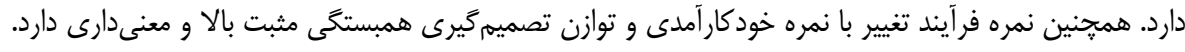

جدول f - متغير هاى بيشگَويى كننده رفتار خودآزمايى پِستان در زنان مورد مطالعه

\begin{tabular}{|c|c|c|c|c|}
\hline Wald & معنى ماربودن & نسبت شانس (فاصله اطمينان ه9٪\%) & متغير ييشكَويى كننده & متغير وابسته(a) \\
\hline & P-Value & OR $(95 \%$ CI $)$ & & \\
\hline 19/8 & $<\cdot 1 \cdot * 1$ & $\cdot / T \wedge(\cdot / 1 q-\cdot / \& q)$ & خود كار آمدى & \multirow{2}{*}{ ييشتفكر } \\
\hline$k / . r$ & $.1 \cdot t^{c}$ & $\cdot / V V(\cdot / 09-\cdot / 99)$ & آَكاهى & \\
\hline Q/Ar & $.1 \cdot r$ & $\cdot \operatorname{lor}(\cdot / \mathrm{MI}-\cdot / 19)$ & خود كار آمدى & \multirow{2}{*}{ تفكر } \\
\hline$r / v$ & 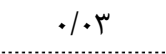 & $\cdot / V \Delta(\cdot / \Delta V-\cdot / 9 V)$ & آكاهى & \\
\hline$r / . q$ & $<\cdot 1 \cdot+1$ & $\cdot / T \Delta(\cdot / / r-\cdot / \kappa \wedge)$ & خود كار آمدى & آَادادَى \\
\hline $.1+.1$ &.$/ 9 V$ & $1 / \cdot F(\cdot / N-N / M r)$ & ابتدايى & \multirow{5}{*}{ عمل } \\
\hline $1 / V^{c}$ & .119 & • & راهنمايى و متوسطه & \\
\hline I/AV & ( & $\cdot|r|\left(\cdot / \cdot \Delta-\cdot \mid \lambda r^{c}\right)$ & تحصيلات & \\
\hline V/TK & $\cdot 1 \cdot \cdot V$ & $\cdot 1 \cdot 1(\cdot / \cdot 1-\cdot / 4 q)$ & فوق دييلم & \\
\hline- & - & - & ليسانس و بالاتر & \\
\hline
\end{tabular}

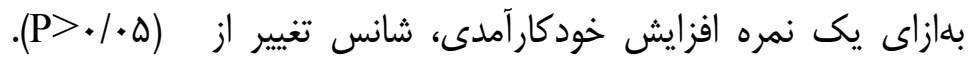
مرحله ييشتفكر به مرحله نكَهدارى 1// • افزايش ييدا كرد.

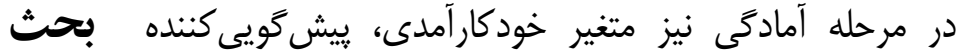

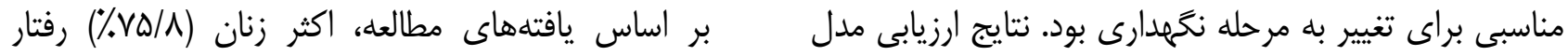

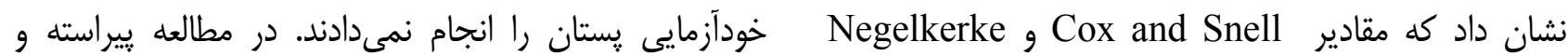

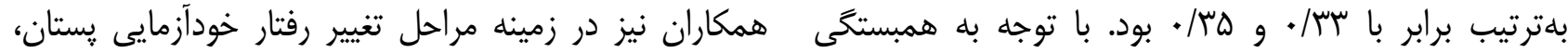

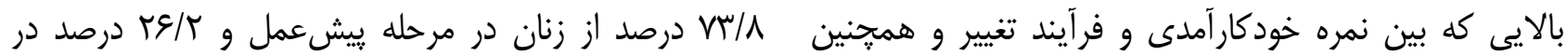
نمره توازن تصميمگيرى و فرآيند تغيير وجود داشت، متغيير مرحله عمل قرار داشتند (19). در مطالعه واحديان و همكاران

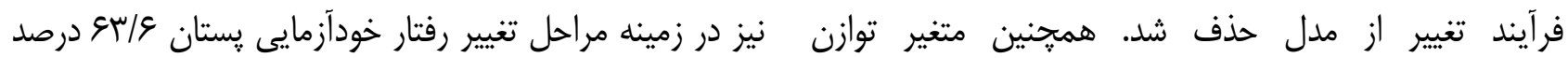

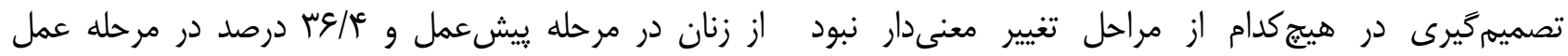


انخَيزه مىتواند انجام عمل را افزايش يا كاهش دهد. افرادى

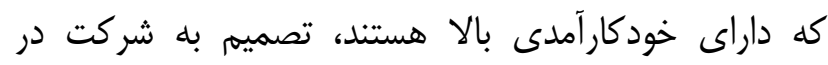

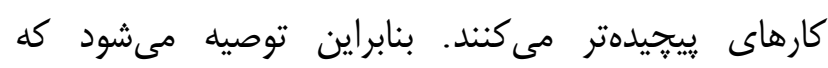

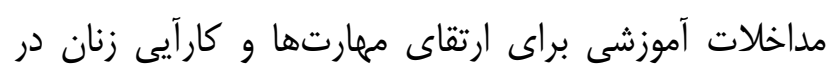
مورد خودآزمايى يستان طراحى و اجرا شود.

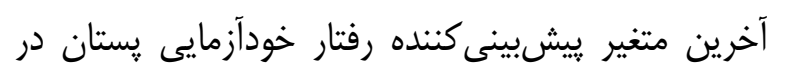

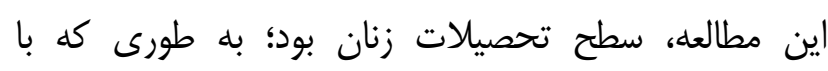

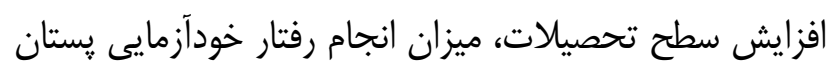
نيز افزايش يافت. نتايج مطالعه حاضر در اين زمينه با مطالعات

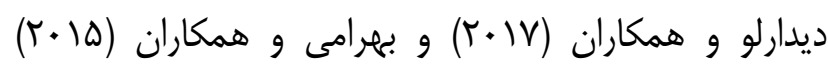

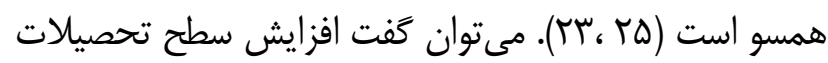

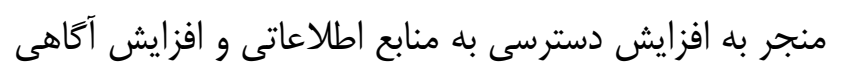

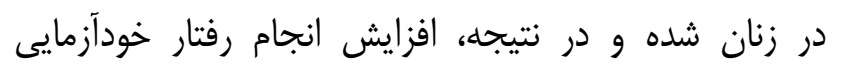
يستان را منجر مىشود.

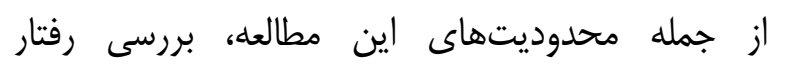

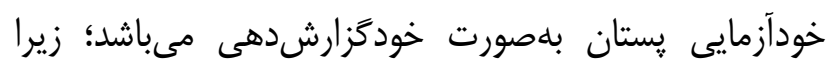

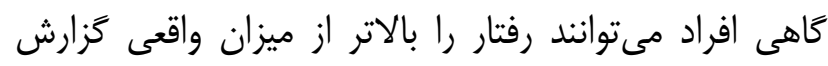

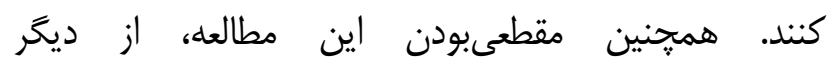

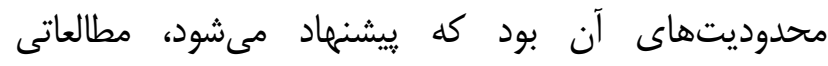
بلهورت مداخلهاى در اين زمينه طراحى و اجرا شود.

\section{نتيجه كيرى}

از يافتههاى اين مطالعه مئوتوان نتيجه كرفت كه با

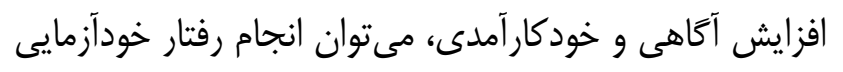

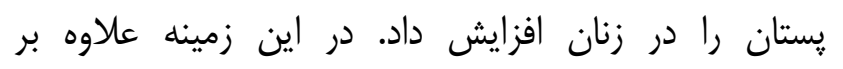

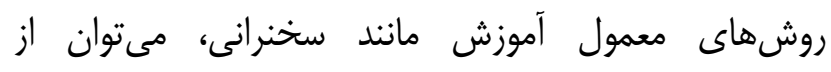

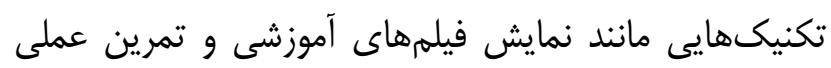

$$
\text { بر روى ماكت بهره كرفت. }
$$

\section{تقدير و تشكر}

نويسندًان مقاله مراتب تقدير و تشكر خود را از معاونت

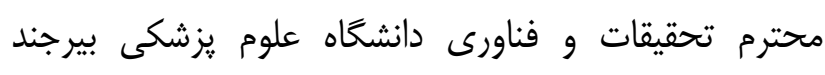

قرار داشتند (•r). در مطالعه Redhwan و همكاران (II (r)

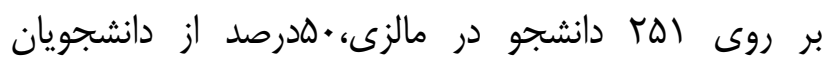

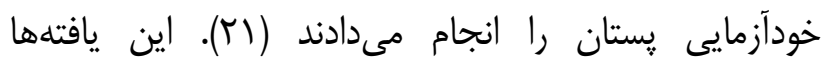

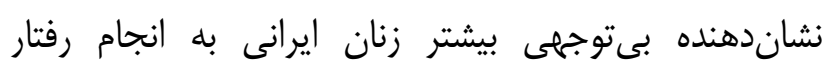

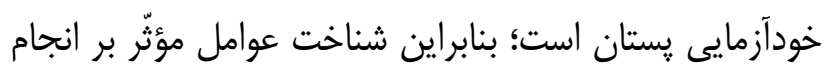

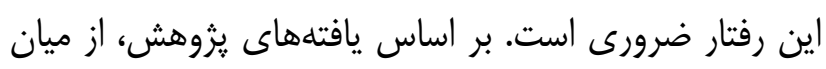

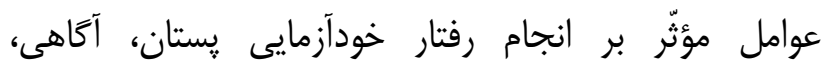

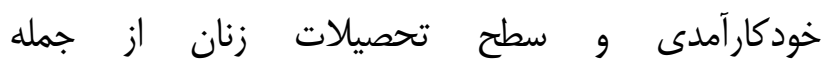

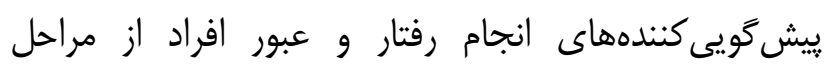
ييش عمل به مراحل عمل بودند.

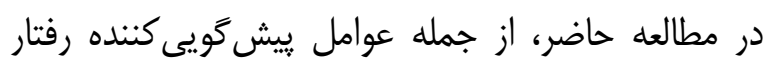

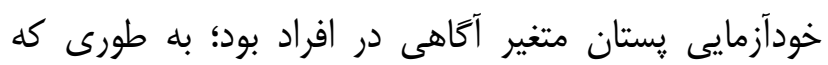

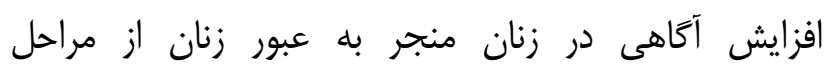

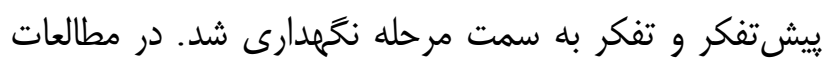

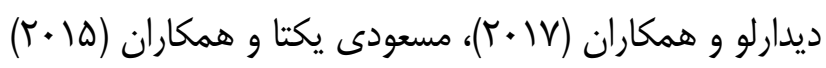

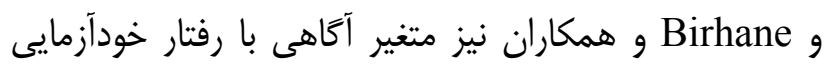
يستان در ارتباط بود؛ به طورى كه در مطالعات نهات آنها كسب

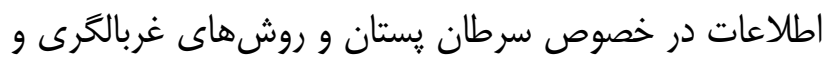

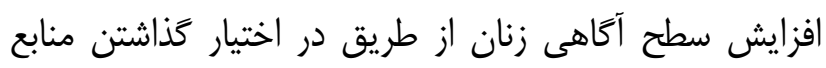

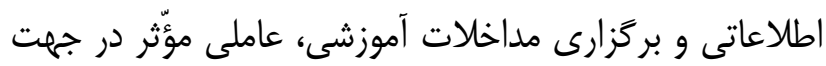

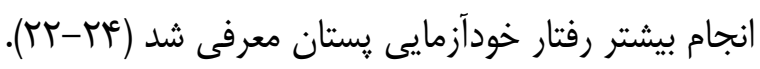

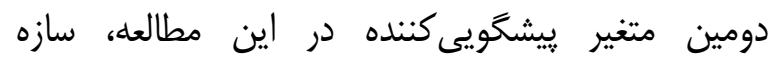

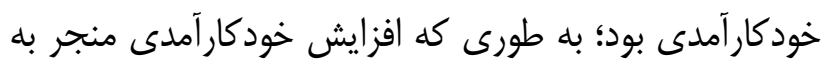

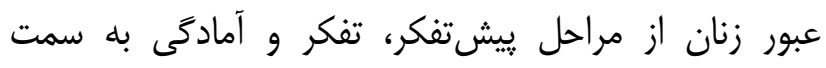

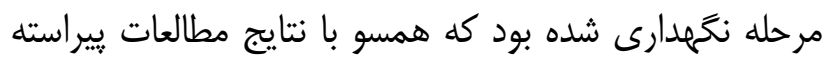

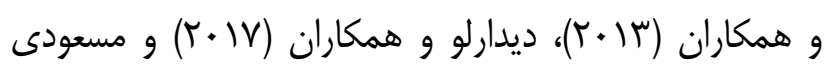

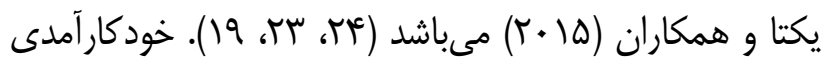

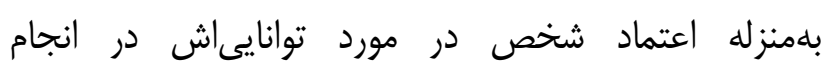

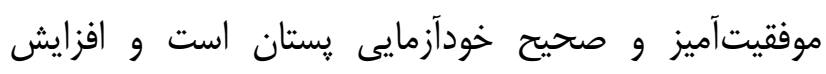

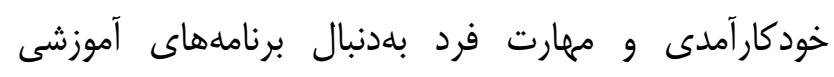

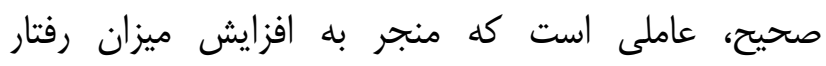

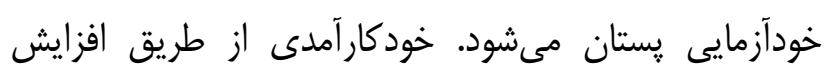




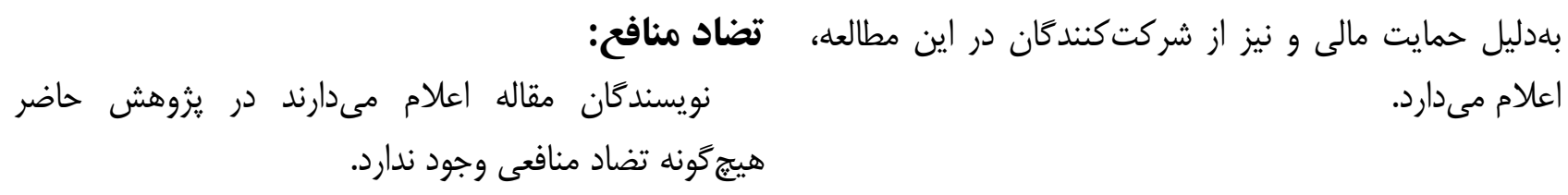

1- Chisti MA, Alfadley AA, Banka N, Ezzat A. Cutaneous Metastasis from Breast Carcinoma: a brief report of a rare variant and proposed morphological classification. Gulf J Oncolog. 2013; 1(14): 90-4.

2- Moodi M, Norozi E, Rezaeian M, Mostafavi F, Sharifirad Gh. Comparing the effectiveness of three educational interventions based on health belief model on mammography screening behaviors in women above 40 years in Isfahan, Iran. J Health Syst Res. 2013; 9(4): 385-94. [Persian]

3- Bakhtariagdam F, Nourizadeh R, Sahebi L. The Role of Health Belief Model in Promotion of Beliefs and Behaviors of Breast Cancer Screening in Women Referring to Health Care Centers of Tabriz in 2010. Med J Tabriz Univ Med Sci. 2012; 33(6): 25-31. [Persian]

4- Gençtürk N. The status of knowledge and practice of early diagnosis methods for breast cancer by women healthcare professionals. J Breast Health. 2013; 9(1): 5-9.

5- Sarfo LA, Awuah-Peasah D, Acheampong E, Asamoah F. Knowledge, attitude, and practice of self-breast examination among female university students at Presbyterian University College, Ghana. Am J Res Commun. 2013; 1(11): 395-404.

6- Tavafian SS, Hasani L, Aghamolaei T, Zare S, Gregory D. Prediction of breast self-examination in a sample of Iranian women: an application of the Health Belief Model. BMC Womens Health. 2009; 9: 37.

7- Anwar SL, Tampubolon G, Van Hemelrijck M, Hutajulu SH, Watkins J, Wulaningsih W; PILAR Research Network. Determinants of cancer screening awareness and participation among Indonesian women. BMC Cancer. 2018; 18(1): 208.

8- Gonzales A, Alzaatreh M, Mari M, Saleh AM, Alloubani A. Beliefs and Behavior of Saudi Women in the University of Tabuk Toward Breast Self-Examination Practice. Asian Pac J Cancer Prev. 2018; 19(1): 121-6.

9- Badakhsh M, Balouchi A, Taheri T, Bouya S, Ahmadidarehsima S, Aminifard MN. Attitude and Practice Regarding Breast Cancer Early Detection among Iranian Women: A Systematic Review. Asian Pac J Cancer Prev. 2018; 19(1): 916.

10- Pilevarzadeh M, Ahmadidarrehsima S, Salari S. Women's Perception of Barriers to Breast Self-examination (BSE). Prensa Med Argent. 2018; 104: 5.

11- Early J, Armstrong SN, Burke S, Thompson DL. US female college students' breast health knowledge, attitudes, and determinants of screening practices: new implications for health education. J Am Coll Health. 2011; 59(7): 640-7.

12- Menezes MC, Mingoti SA, Cardoso CS, Mendonça Rde D, Lopes AC. Intervention based on Transtheoretical Model promotes anthropometric and nutritional improvements-A randomized controlled trial. Eat Behav. 2015; 17 : 37-44.

13- Glanz K, Rimer B, Viswanath K. Health behavior: Theory, research, and practice. San Francisco: Jossey-Bass Inc Pub; 2015.

14- Saffari MS, Hojaeezade H, Ghofrani Pour F, Heydarnia A, Pakpor Hajiagha A. Heories, models and methods of health education and health promotion. $1^{\text {st }}$ ed. Tehran: Asare Sobhan Publication; 2009. [Persian]

15- Mostafavi F, Ghofranipour F, Feizi A, Pirzadeh A. Improving physical activity and metabolic syndrome indicators in women: a transtheoretical model-based intervention. Int J Prev Med. 2015; 6: 28.

16- Fernandez AC, Amoyal NR, Paiva AL, Prochaska JO. Motivation for HPV Vaccination Among Young Adult Men: Validation of TTM Decisional Balance and Self-Efficacy Constructs. Am J Health Promot. 2016; 30(3): 163-71. 
17- Araban M, Tavafian SS, Zarandi SM, Hidarnia AR, Burri A, Montazeri A. A behavioral strategy to minimize air pollution exposure in pregnant women: a randomized controlled trial. Environ Health Prev Med. 2017; $22: 26$.

18- Whitehead D, Wang Y, Wang J, Zhang J, Sun Z, Xie C. Health promotion and health education practice: nurses' perceptions. J Adv Nurs. 2008; 61(2): 181-7.

19- Pirasteh A, Khajavi Shojaie K, Kholdi N, Davati A. Stages of change and predicting of self efficacy construct in breast self examination behavior among women attending at Tehran Health Centers, Iran, 2011. Iran J Obstet Gynecol Infertil. 2013; 16(70): 16-23.

20- Vahedian Shahroodi M, Pourhaje F, Esmaily H, Pourhaje F. The relationship between breast self-examination and stages of change model in health volunteers. Journal of Research and Health. 2015; 5(1): 13-20.

21- Al-Naggar RA, Al-Naggar DH, Bobryshev YV, Chen R, Assabri A. Practice and barriers toward breast selfexamination among young Malaysian women. Asian Pac J Cancer Prev. 2011; 12(5): 1173-8.

22- Birhane N, Mamo A, Girma E, Asfaw S. Predictors of breast self-examination among female teachers in Ethiopia using health belief model. Arch Public Health. 2015; 73(1): 39.

23- Didarloo A, Nabilou B, Khalkhali HR. Psychosocial predictors of breast self-examination behavior among female students: an application of the health belief model using logistic regression. BMC Public Health. $2017 ; 17(1)$ : 861.

24- Masoudiyekta L, Dashtbozorgi B, Gheibizadeh M, Saki Malehi A, Moradi M. Applying the Health Belief Model in Predicting Breast Cancer Screening Behavior of Women. Jundishapur J Chronic Dis Care. 2015; 4(4): e30234.

25- Bahrami M, Taymoori P, Bahrami A, Farazi E, Farhadifar F. The Prevalence of breast and Cervical Cancer screening and related factors in woman who refereeing to health center of Sanandaj city in 2014. Zanko J Med Sci. 2015; 16(50): 1-12. [Persian] 